\title{
Provider provision of respectful maternity care: Findings from a small scale evaluation in Chipata, Zambia
}

Breakthrough RESEARCH

Follow this and additional works at: https://knowledgecommons.popcouncil.org/departments_sbsr-rh How does access to this work benefit you? Let us know!

\section{Recommended Citation}

Breakthrough RESEARCH. 2020. "Provider provision of respectful maternity care: Findings from a smallscale evaluation in Chipata, Zambia," Programmatic Research Brief. Washington, DC: Population Council. 
USAID-funded SM360+ project and the District Health Office (DHO) of Chipata, Zambia. Findings from the formative research phase contributed to developing solutions through an iterative behavior design process. The design process resulted in the development of a five-component solution package: (1) BETTER Pain Management Toolkit; (2) feedback box; (3) provider-client promise; (4) fresh start funds; and (5) reflection workshop. More details on the designs can be found in Box 1 . This brief outlines key findings and insights into the effect of the solutions on clients' experience of care during labor and delivery.

The theory of change (Figure 1) outlines our understanding of the RMC pathways through provider behavior change, and shows our conceptualization of how our solutions will influence provider behavior, with a focus on the individual enacting the harm and mistreatment.

\section{Research Design}

We conducted a pilot of the RMC solution package in 10 urban and rural facilities in Chipata, Zambia. Facilities were eligible for inclusion if SM360+ operated programs at the facilities. All five components of the solution package were introduced in five facilities, and matched with five comparison facilities based on the following criteria: average number of monthly deliveries, number of staff, size of catchment population, and distance from the DHO (city center).

In September 2019, we collected baseline data at intervention and comparison facilities followed by endline data collection in December 2019. Provider surveys captured providers' perceptions of RMC, including the patient experience and levels of rapport, empathy, and trust that existed between providers and clients. Clients were surveyed four to eight weeks postpartum, as recommended

\section{BOX 1 RMC SOLUTIONS}

Below is a complete list of the five components of the RMC solution package. For more information on the solutions and behavioral barriers addressed, please see our previous programmatic brief and manuscript here.

BETTER Pain Management Toolkit: BETTER stands for Breathe, Encourage, Turn, Think, and Rub. The toolkit includes: (1) BETTER Pain Management technique posters; (2) BETTER Pain Management manual; (3) Massage balls; and (4) BETTER Pain Management partograph guide. The toolkit is intended to incorporate the pain management into routine client care.

Feedback Box: Women were provided with a token upon discharge from the maternity ward and instructed to insert the token into the slot that best reflected their level of satisfaction with the service they received. The feedback box was intended to empower clients to share feedback and provide the means to regularly assess clinic performance.

Provider-Client Promise: The promise sought to clarify and set expectations for behavior of both providers and clients and reassure clients of the treatment they should receive. The document was read aloud by providers upon admission to the labor ward and was signed by both provider and client.

Fresh Start Funds: Facilities were provided with a small fund, which they used to make changes to the labor ward to improve the non-clinical client experience. The funds are intended to generate a sense of "fresh start" for the staff and sense of agency in changing the experience of care.

Reflection Workshop: The workshop encouraged providers to reflect on client care, build an intention to change care as a facility, and introduce solutions. 
FIGURE 1 THEORY OF CHANGE

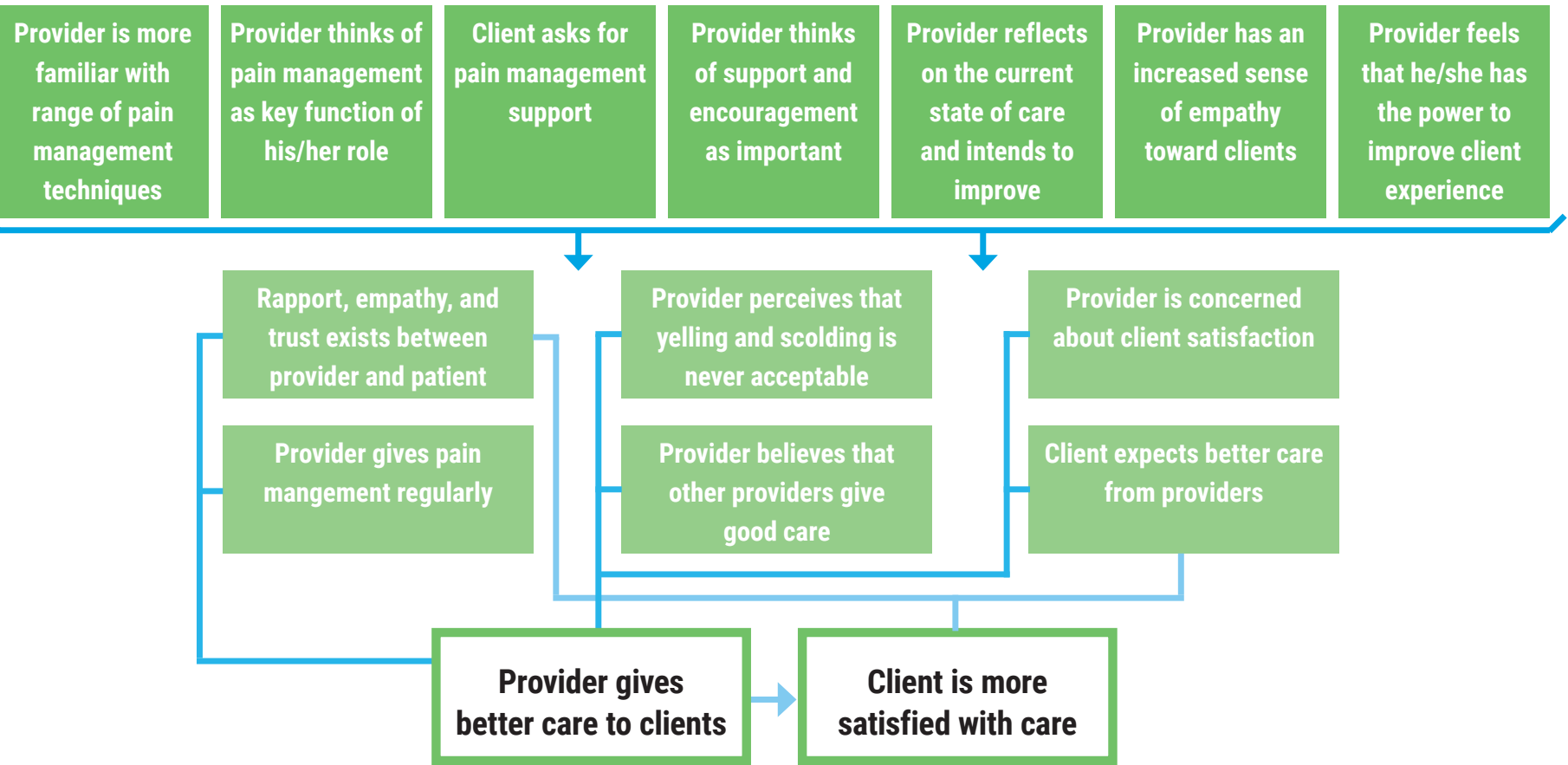

by the RMC literature in order to most accurately capture experiences of disrespect and abuse during labor. ${ }^{3}$ Both providers and clients were also asked open-ended qualitative questions at endline on perceptions of care and of the solution package. We also conducted monitoring visits and interviewed facility in-charges in the implementation facilities over the course of the implementation period. Tables 1 and 2 provide an overview of survey participants.

We assessed the effectiveness of the RMC solution package on the outcomes described in the theory of change to determine whether the interventions improved client experience of care during facility-based deliveries. We measured the differences in outcomes amongst intervention and comparison groups and controlled for certain variables at endline. ${ }^{\mathrm{b}}$ Because we were not able to randomly assign facilities to intervention or comparison groups, an additional sensitivity analysis was also conducted to validate the findings. In instances where the sensitivity analysis results did not validate findings, we believe there was a meaningful difference in the outcome amongst comparision and intervention, though the size of the effect may be less known due to factors we could

${ }^{b}$ In the provider analysis we controlled for the outcome variable at baseline, whether a provider was a midwife, provider's gender, years of experience attending deliveries, and number of deliveries within the last two weeks. Client-level analysis controls included facility-level baseline averages for each outcome of interest, client's marital status, age, and parity. not control for. Qualitative data was also used to develop a more nuanced understanding of the results. The results described in this brief are statistically significant or marginally significant at a $p<0.10$.

\section{TABLE 1 DEMOGRAPHICS OF PROVIDERS SURVEYED}

\begin{tabular}{|lcc|}
\hline Providers & $\begin{array}{c}\text { Baseline } \\
(\mathbf{n}=33)\end{array}$ & $\begin{array}{c}\text { Endline } \\
(\mathbf{n}=35)\end{array}$ \\
\hline Gender & $85 \%$ female & $77 \%$ female \\
Cadre & $55 \%$ midwives & $54 \%$ midwives \\
$\begin{array}{lcc}\text { Average years of experience } \\
\text { attending deliveries }\end{array}$ & 9.7 & 9.3 \\
\hline
\end{tabular}

\section{TABLE 2 DEMOGRAPHICS OF CLIENTS SURVEYED}

\begin{tabular}{|lcc|}
\hline Clients & $\begin{array}{c}\text { Baseline } \\
(\mathrm{n}=60)\end{array}$ & $\begin{array}{c}\text { Endline } \\
(\mathrm{n}=92)\end{array}$ \\
\hline Age & 23.5 years old & 24.5 years old \\
\hline Marital status & $95 \%$ married & $79 \%$ married \\
Average parity & 2.5 children & 2.4 children \\
$\begin{array}{l}\text { Average time since delivery } \\
\text { at time of survey }\end{array}$ & 1 month & 1.1 months \\
\hline
\end{tabular}




\section{Key Findings}

The findings below focus on evidence of the effectiveness of the intervention package and highlight additional lessons learned that pertain to RMC and client experience more broadly.

\section{Disrespect and abuse}

\section{Clients were less likely to experience disrespect and abuse.}

Evidence suggests that at endline, clients at implementation facilities were significantly less likely (15 percentage points) to experience any form of disrespect and abuse compared to clients at comparison facilities as reported by clients. Types of disrespect and abuse reported across client types at endline included lack of privacy, threats, delivering alone and feeling abandoned, and being made to feel uncomfortable. Perhaps because instances of disrespect were quite low at intervention facilities, the sensitivity analysis did not confirm these findings and given the limited sample it may not be possible to distinguish such small changes in outcomes.

There were no observed differences in witnessing or engaging in acts of disrespect and abuse from the perspective of providers.

At endline, providers at intervention facilities were no more likely to ever witness disrespect and abuse compared to providers at comparison facilities. Providers at both intervention and comparision facilities reported witnessing all four kinds of disrespect and abuse explored (use of force, threatening client, showing disrespect due to client attribute, and scolding), though scolding was the most commonly reported form. Despite high rates of witnessing disrespectful care, providers generally reported that their colleagues treated clients acceptably. A much smaller percentage of intervention and comparison providers shared that they themselves had done anything disrespectful, and there was no significant difference between intervention and comparison providers in self-reported acts of disrespect and abuse.

Evidence on whether there was a change in provider's perception that yelling or scolding is never acceptable is not clear.

Marginally significant findings at endline suggest that providers at the intervention facilities were more likely to state that providers at their facility believe that yelling at or scolding a patient is never acceptable compared to

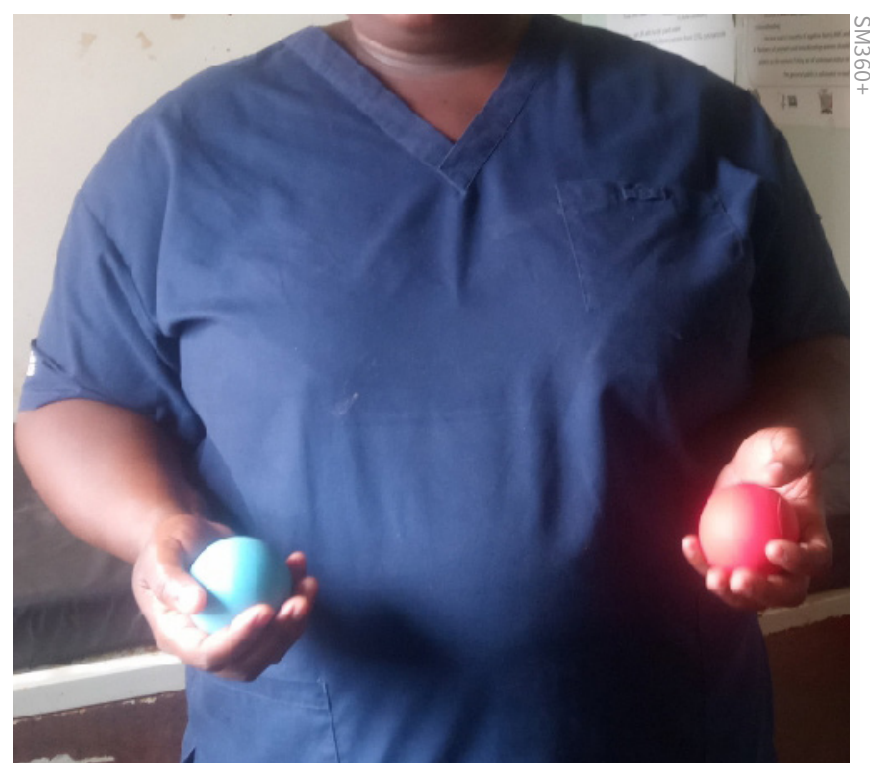

providers at comparison facilities; the findings were not confirmed by sensitivity analysis. Results suggest that while some providers do not condone yelling, others may still justify scolding a patient under certain circumstances.

\section{Provider burnout was low and was not linked to reported engagement in disrespectful care.}

The Maslach Burnout Inventory section on empathy and dehumanization ${ }^{c}$ was adapted and applied, and survey results found low levels of burnout at baseline across providers. We also found that there was no correlation between provider burnout and self-reported instances of disrespectful care. This result aligns with findings from our formative research which suggested that burnout was not a key driver of disrespectful care in this context.

\section{Provision of pain management}

\section{Clients were more likely to request pain management support.}

We asked clients whether they asked the provider for help when they were feeling pain. Evidence suggests that clients at intervention facilities were significantly more likely to request pain management compared to clients who delivered at comparison facilities; 77 percent of clients at intervention facilities as compared to 36 percent of clients at comparison facilities. These results were confirmed by the sensitivity analysis.

'The Maslach Burnout Inventory is typically used to self-assess level of burnout. A subset of the questions was modified and adapted for use in our survey, specifically to measure burnout as it relates to dehumanization and empathy. 
Providers were more likely to see pain management as a key function of their role.

We asked providers to select the three most important tasks they perform during delivery from a pre-determined list of common tasks based on our formative research. Findings were marginally significant suggesting that providers at intervention facilities were more likely to rate pain management as one of the most important tasks during delivery compared to providers at comparison facilities at endline; 23 percent of providers at intervention facilities compared to 8 percent of comparison providers. Though our sensitivity analysis did not confirm the results, our qualitative findings also suggest that the intervention had a meaningful effect. For instance, providers shared that the RMC solutions helped emphasize their responsibility to provide pain management. They also mentioned that the provider-client promise served as another reminder of the importance of providing pain management.

\section{Providers shifted the types of pain management tech-} niques used.

We observed a shift in the types of techniques used amongst intervention providers from baseline to endline. At baseline the most commonly cited techniques used by providers when a client requested pain management were massage, encouragement, and chat. At endline, the three most commonly applied techniques were massage, breathing exercises, and changing of position, which were all techniques outlined in the BETTER Pain Management Toolkit

Moreover, several clients noted the use of the massage ball as something that they particularly enjoyed and something different to previous deliveries.

\section{I loved the way they treated me and the use of a ball to rub my back, the way they used to talk to me when in pain, and the way they encouraged me.}

-Client delivering at intervention facility

\section{Agency to improve quality of care}

Perceived agency to improve quality of care among all providers was high at baseline, and did not increase at endline.

While all providers, in both intervention and comparison facilities, noted that they were very or extremely interested in improving care at facilities at baseline, most

\section{FIGURE 2 TYPES OF PAIN MANAGEMENT TECHNIQUES USED DURING LABOR AND DELIVERY BY INTERVENTION PROVIDERS}
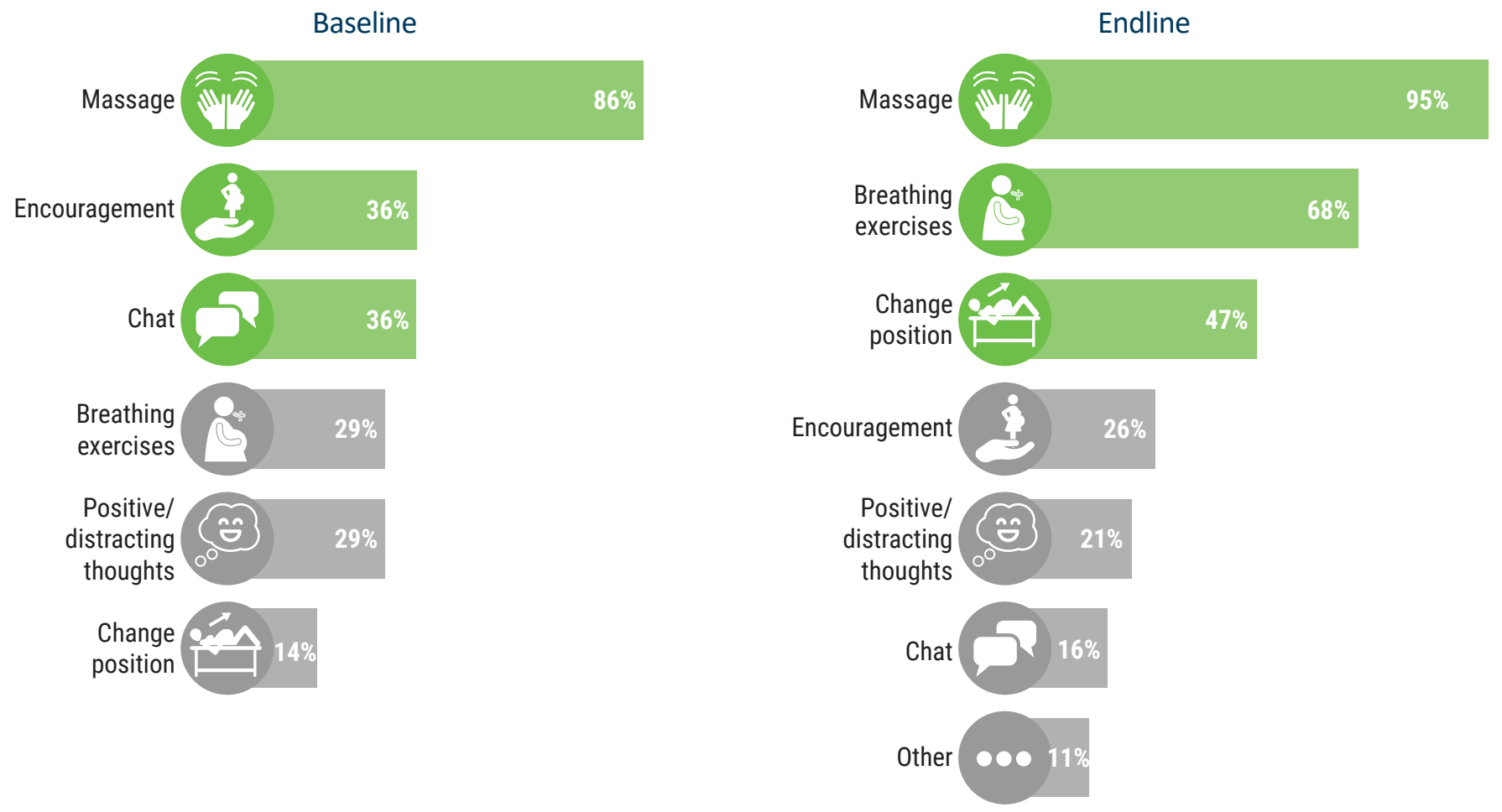
providers at intervention and comparison facilities did not report a need to improve.

When asked to describe the state of care of their facility, most providers across intervention and comparison facilities, evaluated the state of care favorably. It appears that providers already feel confident in their ability to make a change and that the "fresh start" funds were not necessary to contribute to enhancing a sense of agency amongst providers. While other solutions may also influence provider agency, it was not their primary objective.

\section{Rapport, empathy, and trust between provider and patient}

\section{Providers were more likely to be empathetic toward cli- ents and agree that clients are cooperative.}

Evidence suggests that at endline, providers at intervention facilities were significantly more likely to strongly agree that clients are often cooperative during labor and delivery compared to providers at comparison facilities. Results were also marginally significant and suggested that providers in intervention facilities were more likely to be empathetic toward clients. Both sets of results were however, not corroborated by the sensitivity analysis.

\section{Clients reported high levels of trust in providers, though qualitative findings showed some variation.}

At both baseline and endline, almost all clients reported trusting their provider, feeling that their provider cared for them, and believing that their satisfaction was important to providers. Similarly, almost all providers reported that client satisfaction was important or very important to their work. However, clients' qualitative reflections and results from other quantitative questions were mixed as described in the following section. For instance, several clients described feeling a sense of relief at being promised the kind of care described in the provider-client promise, thus indicating that not all clients felt confident in the care they would receive otherwise.

Clients reported that they felt confident that the provider would follow their promise, and none reported feeling that the promise had been broken during her delivery. Clients also remarked that the promise was educational and that they valued being consulted and involved. These comments suggest that while clients may report high levels of trust in providers already, they also perceive value in the promise as a means of reassurance of the care they will receive.

\section{Client expectations and satisfaction}

While clients reported being satisfied with care, clients' expectations for respectful care were low and did not increase during implementation.

At baseline, across intervention and comparison facilities, almost half of clients said they expected that a provider would yell or scold her and one-third said they expected the provider might use insults, intimidations, threats, or coercion. Several women explicitly mentioned that they expected to be shouted at or slapped either because they were arriving late to the facility or because this is what they had heard from others.

Despite having an expectation of disrespectful care, almost all clients, across intervention and comparison facilities, also reported an expectation that providers would provide "good care." This suggests that clients think of "good care" in terms of clinical care rather than respectful care. Not being shouted at, beaten, or the provider's assistance with anything not immediately essential to a safe delivery (such as helping to clean blood after delivery) were viewed as reasons to be particularly satisfied with the care received rather than examples of care one should expect. We did not observe any differences in expectations at endline.

The risk of losing the baby was a main concern for many women and was likely the primary driver of reported satisfaction with delivery.

Across intervention and comparison facilities, the qualitative findings suggest that women's low expectations of interpersonal care may be linked to their focus on the baby's survival. Several women explained that they perceived a real risk that the baby might not survive and allowed themselves to develop feelings for the baby only once they had the certainty that the baby would live. Clients also shared that their primary concern during delivery was delivering a healthy baby and our qualitative data suggested that even when clients expected disrespect and abuse, they reported being satisfied by the care they received since they delivered a healthy baby.

Clients expressed confidence in the feedback box and providers also reported its utility in understanding client satisfaction.

While there was no detectable impact in client satisfaction or its importance to providers, qualitative results suggest that both clients and providers at intervention facilities found utility in the feedback box. Providers described the 
feedback box as a means to understand client satisfaction; as one provider shared,

\begin{abstract}
[F]or example we are having unsatisfied clients, it will help us look into the matter and see where we are having the problem. If the clients are very satisfied and we have a lot of tokens then we know that we are doing our job and clients are appreciating ...one or two things that they are not happy about, we try to talk among ourselves and try to solve the issue so that all the mothers can go home happy.... Yes, health workers are working very hard so that every mother is very satisfied. We don't want to have any satisfied and unsatisfied mothers.
\end{abstract}

-Provider at intervention facility

Clients from intervention facilities, remarked "feeling good" about being asked to share their level of satisfaction through the feedback box. Additionally, clients commonly noted that they believed that positive feedback would be motivational for providers and that negative feedback would lead providers to change, thus suggesting their confidence in the feedback mechanism.

\section{The Case for Further Research}

The results of this small-scale study act as a proof of concept-showing that the solution package can be implemented effectively and providing early evidence of potential for impact. These results support the case for a larger scale evaluation to further validate the effectiveness of the solutions and identify the relative effects of the five components of the solution package. While the "Fresh Start" funds may not have been necessary to increase levels of provider agency, which were already high at baseline, we would not exclude them from furture research if appropriate for the context.

While these early results are promising, the study faced a number of limitations related to its study design and short duration. The limitations of this study, when combined with its promising results, support the case for further research. Research at a larger scale could more rigorously test impact, deepen understanding of the effectiveness of the solutions, and inform ongoing refinements to the solution package to strengthen their impact on quality and client experience of care. Additionally, larger scale research could better inform future scaling efforts by generating insights around how solutions can be adapted to ensure impact at scale and in different contexts.

\section{Promise for Impact}

This small-scale evaluation provides promising evidence of the potential for behaviorally-informed solutions to increase provision of RMC. While we were unable to parse out the relative effects of the five components of the solution package, our results suggest that all but one appear to have contributed meaningfully to these positive results. The fresh start funds were intended to increase providers' perceived agency to make positive changes for clients and do not appear to have had this effect in Chipata-likely because perceived agency was already high among this population of providers at baseline. The other four components of the solution package-the reflection workshop, Pain Management Toolkit, provider-client promise, and feedback box-all appear to have contributed to the positive results.

Additionally, as described here as well as in our implementation brief, providers were able to successfully implement the solution package, and both providers and clients valued it as a means to enhance their work or delivery experience. We are encouraged by the results and together with SM360+ will engage with officials from the DHO of Chipata, as well as the Ministry of Health to determine how these promising results could be leveraged to inform RMC programming.

These early results demonstrate that behaviorally-informed solutions can improve provision of RMC, quality of care, and client satisfaction. In other settings where providers face barriers to RMC similar to those identified in our formative research, an adaptation of this solution package might lead to similarly positive results. Programmers interested in adapting and replicating the package are invited to contact the research team. 


\section{References}

${ }^{1}$ WHO Reproductive Health Library. WHO recommendation on respectful maternity care. (February 2018). The WHO Reproductive Health Library; Geneva:

World Health Organization

${ }^{2}$ Bohren, Meghan A. et al. 2019. "How women are treated during facility-based childbirth in four countries: a cross-sectional study with labour observations and community-based surveys." The Lancet 394(10210): 1750-63.

${ }^{3}$ Kruk, Margaret E. et al. 2018. "Disrespectful and abusive treatment during facility delivery in Tanzania: a facility and community survey." Health Policy and Planning 33(1): e26-33.

\section{Acknowledgments}

This research brief describes work led by ideas 42 under Breakthrough RESEARCH. This brief and the work it describes is possible through the work and support of SafeMotherhood360+, Population Council Zambia, and Population Council, Washington, DC

\section{Suggested citation:}

Breakthrough RESEARCH. 2020. "Provider provision of respectful maternity care: findings from a small-scale evaluation in Chipata, Zambia," Programmatic Research Brief. Washington, DC: Population Council.

Cover photo credit: ideas 42

(C2020 The Population Council. All rights reserved.
Email

BreakthroughResearch@popcouncil.org

\section{Breakthrough RESEARCH | Population Council}

4301 Connecticut Ave., NW, Suite 280 | Washington, DC 20008

+12022379400 | breakthroughactionandresearch.org

\section{POPULATION} COUNCIL

Ideas. Evidence. Impact.

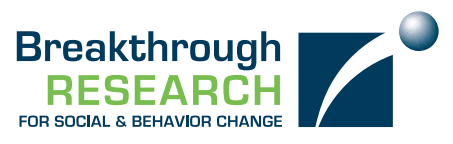

Breakthrough RESEARCH is made possible by the generous support of the American people through the United States Agency for International Development (USAID) under the terms of cooperative agreement no. AIDOAA-A-17-00018. The contents of this document are the sole responsibility of Breakthrough RESEARCH and Population Council and do not necessarily reflect the views of USAID or the United States Government.

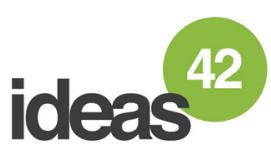

Breakthrough RESEARCH catalyzes social and behavior change (SBC) by conducting state-ofthe-art research and evaluation and promoting evidence-based solutions to improve health and development programs around the world. Breakthrough RESEARCH is a consortium led by the Population Council in partnership with Avenir Health, ideas42, Institute for Reproductive Health at Georgetown University, Population Reference Bureau, and Tulane University. 